\title{
From the image towards a new concept
}

\author{
Lajos Lakatos ${ }^{1}$ - György Balla ${ }^{2}$
}

Received: 12 January 2016 / Accepted: 1 March 2016/Published online: 9 March 2016

(C) Springer Science+Business Media New York 2016

\section{Comment}

Marano M, Gentilucci UV, Altamura C, et al. Altered metal metabolism in patients with $\mathrm{HCV}$-related cirrhosis and hepatic encephalopathy. Metabolic Brain Disease. 2015; 30: 14451452.

It is really exciting in this pre-cited article that dysfunctional metal homeostasis contributes to oxidative stress and neuronal damage in hepatic encephalopathy.

Over the past two decades there have been significant advances in our understanding of metal homeostasis and of neurodegenerative and neurodevelopmental diseases.

The neonatal brain forms and develops over a long period compared to other organs, with neuron proliferation and migration. The blood-brain barrier is not fully developed until the middle of the first year of life. In infants that had have significant exposure to toxic metals, the concentration of the metal is often higher in the fetus than in maternal blood.

Our new hypothesis - in harmony with the work of Marano et al. - addresses the medical necessity of chelation therapy (with D-Penicillamine - D-PA) in the neonatal period

Lajos Lakatos

lakatos1@kenezykorhaz.hu

György Balla

balla@med.unideb.hu

1 Department of Pediatrics, Kenezy Teaching Hospital, Bartók B. str. 2-26, Debrecen 4031, Hungary

2 Department of Pediatrics, University of Debrecen, Egyetem tér 1, Debrecen 4032, Hungary
(Lakatos et al. 2015) as it is feasible that unconjugated bilirubin (UCB) molecule possesses particular affinity to copper stored in basal ganglia where copper-bilirubin complex can be formed together with the production of hydroxyl radical (Farhan et al. 2001). Therefore, the bilirubin-induced neurodevelopmental dysfunction (BIND) is a neurological damage of immature brain caused by accumulation of free metals and UCB-Cu complex (as prooxidant) in the basal ganglia and other parts of central nervous system relevant to BIND. The main comorbidity is the hemolysis. During this process a great amount of heavy metals (mainly iron and copper) may circulate in the bloodstream, and can pass through the blood-brain barrier. UCB has a special affinity for the globus pallidus (Shapiro et al. 2006), the hippocampus, and the subthalamic nucleus because they have a high divalent metal content, mainly iron and copper. In addition UCB can displace metals (copper) from the albumin binding because it binds stronger to albumin than copper (Jianjun et al. 2005). Figure 1. demonstrates our concept about BIND based on the above described hypothesis. Our recently published case reports (Lakatos et al. 2015) and other healthy and highly educated patients of the long-term (28-42 years) follow-up suggest that D-PA therapy of newborn infants may have significant neuroprotective effects in cases jeopardized by BIND or retinopathy of prematurity.

The idea that D-PA treatment of the newborn might be a suitable therapy for control icterus neonatorum occurred, serendipituously, to one of us [L.L.] > 40 years ago, while reflecting on the similarity of copper storage in Wilson's disease and neonates. DPA not only chelates copper from tissue, but also detoxifies tissue copper by promoting the synthesis of metallothionein, which forms a non-toxic combination with copper.

Considering the most important ,discovery” of our D-PAproject we can say that this drug should be undoubtedly 
BIND

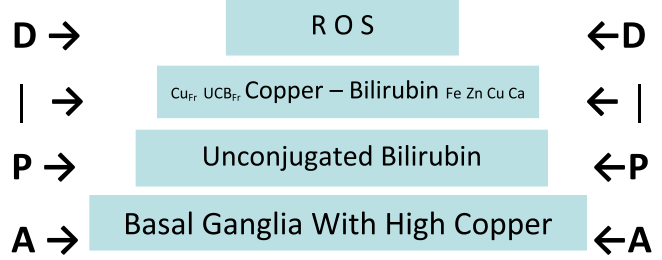

Fig. 1 Potential pathomechanisms of bilirubin-induced (metal mediated) neurodevelopmental dysfunction (BIND), and effects of D-Penicillamine (D-PA) in the neonatal hyperbilirubinemia $(\mathrm{ROS}=$ reactive oxygen species; $\mathrm{Cu}_{\mathrm{Fr}}=$ free copper; $\mathrm{UCB}_{\mathrm{Fr}}=$ free bilirubin). Interpretation of D-PA written sideways: $\mathrm{D}=$ direct antioxidant; $\mid=$ scavenge of ROS and chelation of metals; $\mathrm{P}=$ inhibition of heme oxygenase (a decreased production of UCB) only in the newborn period (age related effect); $\mathrm{A}=$ copper chelation

effective (jaundice and lead burden in neonates and may be in the prevention of retinopathy of prematurity - a well-designed, large multicenter randomized controlled trial is required), safe and quite inexpensive (even more for the developing countries!), and it can be used in very high doses in the neonatal period.
This is the case when an idea develops into new concept.

\section{Compliance with ethical standards}

Conflict of interest The authors declare that the research was conducted in the absence of any commercial or financial relationships that could be construed as a potential conflict of interest.

\section{References}

Farhan SF, Singh S, Ahmad A, et al. (2001) Prooxidant and antioxidant activities of bilirubin and its metabolic precursor biliverdin: a structure-activity study. Chem Biol Interact 137:59-74

Jianjun KZ, Wanzh X, Yanbo W, et al. (2005) A kinetic model and its parameter estimation for the process of binding copper to human serum albumin by a voltammetric method. Anal Bioanal Chem 381: $1552-1557$

Lakatos L, Balla G, Pataki I, et al. (2015) D-penicillamine in the neonatal period: case reports. IJMPCR 4:59-63

Shapiro SM, Bhutani VK, Johnson L (2006) Hyperbilirubinemia and kernicterus. Clin Perinatol 33:387-410 